\title{
FERRAMENTA BIM APLICADA NA GESTÃO DOS SERVIÇOS DE MANUTENÇÃO
}

\author{
Aldo Ribeiro de Carvalho - aldo.carvalho@engenharia.ufjf.br \\ Universidade Federal de Juiz de Fora \\ Juiz de Fora - Minas Gerais
}

Maria Teresa Gomes Barbosa - teresa.barbosa@engenharia.ufjf.br

Universidade Federal de Juiz de Fora

Juiz de Fora - Minas Gerais

\section{Resumo}

As edificações são projetadas e executadas de maneira que possam atender as exigências dos usuários que incluem a segurança, o adequado desempenho, a sua durabilidade, dentre outros, proporcionando a satisfação dos usuários que inclui além do conforto, uma adequada relação custo-benefício. Nesse contexto, a ferramenta Building Information Modeling (BIM) possibilita conceber e coordenar os dados e insumos desde fase de projeto até aos serviços de manutenções. O presente trabalho objetiva apresentar a aplicabilidade dessa ferramenta na gestão dos serviços de manutenção corretivas. Para tanto, efetuou-se um estudo de caso, na sala de aula do Núcleo de Ergonomia, Sustentabilidade e Transporte da Faculdade de Engenharia da UFJF. Empregou-se o estudo metrológico do ambiente, representações gráficas nos softwares AutoCAD 2020 e REVIT 2020, bem como exames visuais das manifestações patológicas existentes. Constatou-se que o BIM é uma ferramenta eficaz para auxiliar na elaboração de laudos técnicos, bem como auxiliam na elaboração do programa de serviços de manutenção.

Palavras-chave: BIM. Manifestações Patológicas. Gestão da Manutenção.

\section{THE BIM TOOL APPLIED IN MAINTENANCE SERVICES MANAGEMENT}

\begin{abstract}
:
The buildings are designed and projected can meet the requirements of users, which include safety, adequate performance, durability, among others, providing user satisfaction that includes, in addition to comfort, an adequate low-cost ratio. In this context, the Building Information Modeling (BIM) tool is very important because it makes it possible to design and coordinate data and inputs from the design phase to maintenance services. The present research aims to study the applicability of this tool in the maintenance services management. The case study was carried out in the classroom of the Ergonomics, Sustainability and Transport Center of the Faculty of Engineering at UFJF. The methods study of the environment was used, graphic representations in the AutoCAD 2020 and REVIT 2020
\end{abstract}


software, as well as visual analysis about pathological manifestations. Finally, it was found that BIM is an effective tool to assist in the preparation of technical reports, as well as assist in the preparation of the maintenance services program.

Keywords: BIM. Pathological Manifestation. Maintenance Management.

\section{INTRODUÇÃO}

O procedimento de modelagem da informação da construção consiste num conjunto de tecnologias de projeto e processos produtivos capazes de gerar e gerir dados da edificação ao longo de seu ciclo de vida, possibilitando o uso, concomitante e interativo, entre diversos profissionais envolvidos no empreendimento (EASTMAN et al, 2018; AL-ASHMORI, 2020). Em resumo, é uma ferramenta onde as atividades, em todo o processo construtivo de uma edificação ou parte dela poderá ser gerenciada ao longo da sua vida útil (PENTTILÃ, 2006; HAM et al, 2020) possibilitando avaliar de forma eficiente e precoce diferentes alternativas para soluções de problemas construtivos (SUCCAR; SHER; WILLIAMS, 2012).

No que se refere aos serviços de manutenção a serem realizados durante a vida útil de uma edificação, o BIM (Building Information Modeling) torna-se atraente para os serviços de "Gestão de Manutenção" sendo efetiva na manutenibilidade do empreendimento (SANTOS; CALMON; ALVES, 2018), carecendo de um maior domínio acerca da potencialidade dessa ferramenta para o setor da construção civil (SENA et al, 2018).

Cabe mencionar que NBR 5674/ 2012 define a manutenção de edifícios como um conjunto de procedimentos que visam conservar ou recuperar a funcionalidade de uma edificação e de todas suas partes constituintes a fim de garantir a segurança para os usuários, sendo identificada, por Pinto e Nascif (2012), em 4 categorias, a saber:

i) Preventiva que obedece a um plano de manutenção, com intervalos bem demarcados, reduzindo ou evitando falhas e quedas de desempenho;

ii) Preditiva objetiva prevenir falhas no sistema, através do acompanhamento contínuo de diversos parâmetros, logo as intervenções são realizadas apenas quando necessário;

iii) Detectiva relacionada à capacidade de detectar, identificar falhas ocultas ou não perceptíveis à operação ou à equipe de manutenção;

iv) Corretiva atua em sistemas construtivos com defeito ou desempenho diferente do esperado, sendo necessário, portanto, a ocorrência de uma falha no sistema construtivo.

Nesse contexto, salienta-se que as manifestações patológicas, normalmente, estão associadas a pelo menos um dos fatores: intrínsecos que são inerentes à edificação (incluem as falhas humanas durante a construção e a utilização e as causas naturais) e/ ou extrínsecos (que possuem origem externa à edificação como, por exemplo, falhas humanas durante o projeto e a utilização e as ações químicas, biológicas e mecânicas) (SOUZA; RIPPER, 1998).

Entretanto, as etapas de planejamento e projeto são vulneráveis e podem onerar a execução e a manutenção de um empreendimento (FOUNTAIN; LANGAR, 2018; DE OLIVEIRA, 2019). Nesse contexto, o diagnóstico das manifestações patológicas é realizado empregando diversos métodos e equipamentos, iniciando por uma minuciosa análise visual e, posteriormente, usando testes não destrutivos ou destrutivos nos materiais e componentes das edificações (CARVALHO et al, 2020). A ferramenta BIM e seus recursos se destacam pelo auxílio à gestão dos serviços de manutenção (VALERO et al, 2019) já que possibilita o armazenamento de dados acerca do processo construtivo e sua consulta torna-se eficaz e de suma importância na elaboração de laudo técnico.

Nesse contexto, o presente trabalho efetua uma análise da aplicação da ferramenta BIM na Gestão de Manutenção em uma obra pública, através de um breve estudo de caso, bem como desenvolve um programa de manutenção corretiva e preventiva. 


\section{MATERIAIS E MÉTODOS}

O estudo de caso trata-se de um As Built da sala de aula do Núcleo de Ergonomia, Sustentabilidade e Transportes (NEST) localizado na Faculdade de Engenharia da UFJF, onde o "como construído" consiste numa metodologia regulamentada pela NBR 14.645/2011 que possibilita a implantação e realização de diversas etapas de controle dimensional de um empreendimento edificado, desde a terraplenagem até a instalação dos sistemas da edificação.

Salienta-se que a Escola de Engenharia foi fundada em 1914 e localizava-se no Centro de Juiz de Fora, na rua Halfeld, $\mathrm{n}^{\circ}$ 175-A. Em 1969 o engenheiro-arquiteto Arthur Arcuri desenvolveu o Campus da Universidade Federal de Juiz de Fora (UFJF) e no ano de 1973 a Escola de Engenharia migrou para as instalações atuais para o campus da UFJF, tornando-se a Faculdade de Engenharia da UFJF (vide Figura 1).

Figura 1 - Faculdade de Engenharia da UFJF

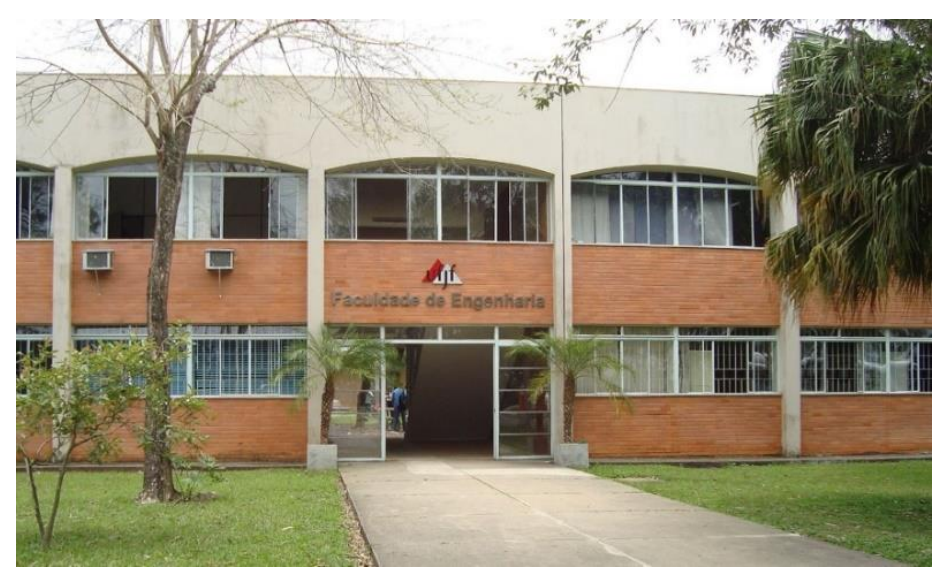

Fonte - Os Autores

Nesse contexto, histórico e construtivo, tem-se, atualmente, a sala do Núcleo de Ergonomia, Sustentabilidade e Transportes, que se dedica ao estudo de gestão, materiais sustentáveis e inovações, manutenção de edificações e transportes.

Inicialmente efetuou-se uma vistoria no local para identificar as manifestações patológicas e, a seguir, no estudo metrológico formalizou-se a representação em planta baixa com auxílio da ferramenta computacional AutoCad 2020 e, por fim, a representação 3D através de um software BIM, o REVIT 2020 da Autodesk. Nas Figuras 2 e 3 é apresentada a sala de aula do NEST.

Figura 2 - Representação obtida através do estudo metrológico (planta baixa da sala de aula)

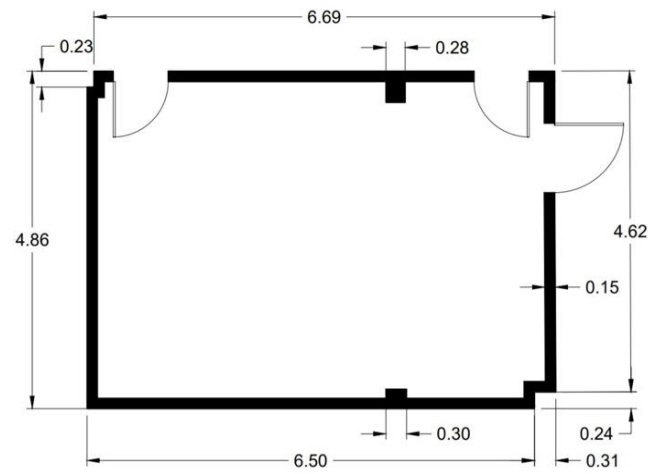

Fonte - Os Autores 
Figura 3 - Representação As Built no REVIT (projeto arquitetônico e hidrossanitário do objeto de estudo)

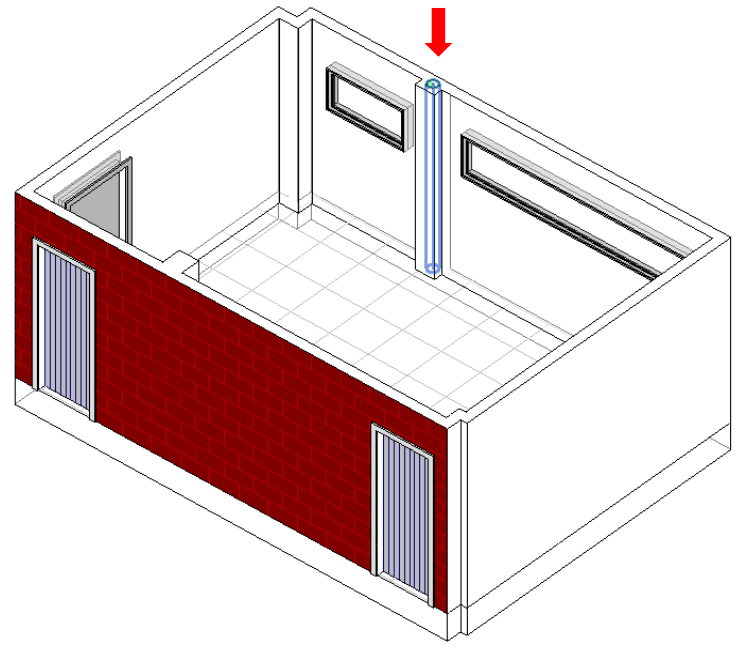

Fonte - Os Autores

Com o As Built realizado, foi possível correlacionar as manifestações patológicas com dados dos sistemas envolvidos no projeto, em especial o sistema hidrossanitário. Além disso, realizou-se a parametrização das informações coletadas com o software BIM.

Para a confecção deste Plano de Manutenção, foi utilizado o método GUT (Gravidade x Urgência x Tendência), que tem como objetivo, em sua primeira etapa, priorizar as ações de intervenção aos problemas de forma racional, seguindo as seguintes etapas: listagem dos problemas ou pontos de análise; pontuação de cada tópico; classificação; tomada de decisões estratégicas (SOTILLE, 2014).

O método GUT prevê para a segunda etapa a atribuição de valores em uma escala de 1 (um) (adotado para problemas menores), à 5 (cinco), considerando o nível de problemas mais complexos, para os critérios de Gravidade (intensidade da ocorrência do dano), Urgência (tempo estimado para solução) e Tendência (padrão evolutivo na ausência de ação), conforme Quadro 1.

Quadro 1 - Critérios de Avaliação para o Método GUT

\begin{tabular}{|c|c|c|c|}
\hline Pontos & Gravidade & Urgência & Tendência \\
\hline 5 & Gravíssimo & Imediatamente & Rapidíssimo \\
\hline 4 & Muito grave & Muito urgente & Muito rápido \\
\hline 3 & Grave & Urgente & Rápido \\
\hline 2 & Pouco grave & Pouco urgente & Pouco rápido \\
\hline 1 & Sem gravidade & Sem urgência & Lento \\
\hline
\end{tabular}

Fonte - adaptado de Sotille (2014) 


\section{RESULTADOS}

Na vistoria realizada à dependência em estudo constatou-se a presença de anomalias no elemento de vedação vertical, parte interna, que ocasionou o desenvolvimento de microrganismos, empolamento da argamassa de revestimento e deterioração da pintura decorrente da presença de umidade acidental (vazamento hidráulico), vide Figuras 4 e 5.

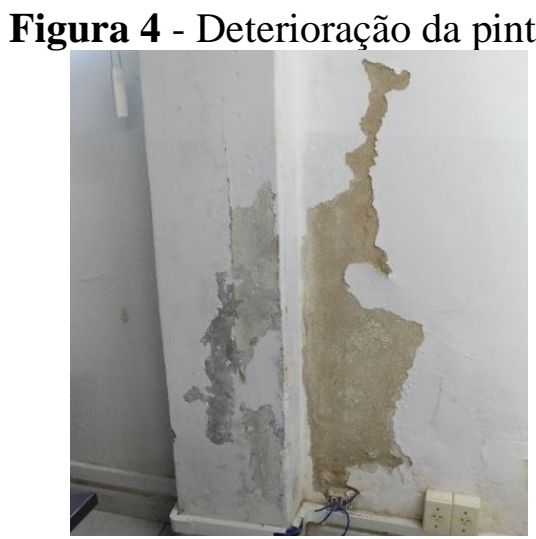

Fonte - Os Autores

Figura 5 - Desenvolvimento de Microorganismos

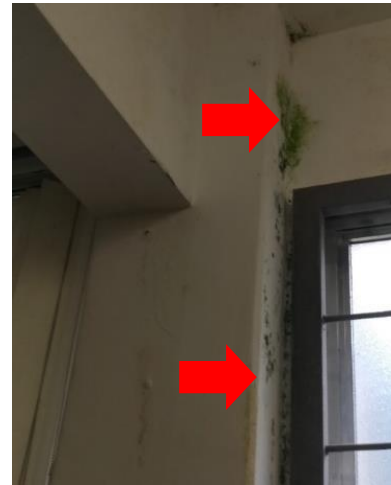

Fonte - Os Autores

Salienta-se que o descolamento com pulverulência da argamassa de revestimento (película de tinta que se descola arrastando o reboco que se desagrega com facilidade) bem como, a proliferação de microrganismos são provenientes de umidade acidental ocasionada pelo vazamento na tubulação hidráulica, que facilita a passagem de fluidos do andar superior para o inferior da edificação. Esse vazamento, por sua vez, foi identificado através das análises conjuntas das informações acerca das manifestações patológicas presentes através do As Built realizado, em ferramenta BIM. Assim, recomenda-se intervenção de forma a sanar os danos existentes na tubulação, seguido de recomposição da argamassa de revestimento, bem como a pintura.

É importante salientar que a presença de umidade e baixa ventilação, favorecem a colonização de microrganismos que são agentes patogênicos para os usuários, o que fortalece a rápida intervenção na região, já que compromete tanto a durabilidade da edificação, bem como o bem-estar dos usuários. Sendo assim, recomenda-se a raspagem das paredes 
deterioradas, recomposição da argamassa de revestimento com aditivos impermeabilizantes e antimicrobianos.

$\mathrm{Na}$ análise de priorização das intervenções correlacionando os diversos fatores que comprometem a durabilidade e vida útil dos empreendimentos, efetuada pelo método GUT, (vide Quadro 2), constata-se que a priorização se evidencia pela segurança e bem-estar do usuário, associada a redução da degradação dos materiais e componentes de construção.

Quadro 2 - Plano de Manutenção

\begin{tabular}{|c|c|c|c|c|}
\hline \multirow{2}{*}{ Manifestação patológica } & \multicolumn{3}{|c|}{ Grau } & \multirow{2}{*}{ Total } \\
\cline { 2 - 4 } & G & U & T & \\
\hline Empolamento e deterioração da pintura (vide Figura 4) & 1 & 2 & 3 & 6 \\
\hline Colonização de Microrganismos (vide Figura 5) & 3 & 3 & 3 & 27 \\
\hline
\end{tabular}

Fonte - Os Autores

A associação dos dados obtidos no modelo BIM ocorreu através da parametrização do método GUT no REVIT, o parâmetro "Patologia" foi desenvolvido, estabelecendo-se prazos para realização dos serviços de manutenção (vide figura 6). A identificação da anomalia, através do software BIM, indicou por meio dos elementos gráficos específico, os componentes da edificação que necessitam de reparos, enquanto as planilhas do REVIT forneceram um documento, concentrando quais são as patologias ocorridas e o tempo máximo para as intervenções necessárias, conforme figura 7.

Figura 6 - Parâmetro Patologia com descrição e tempo necessário estipulado

\begin{tabular}{|c|c|c|c|}
\hline \multicolumn{3}{|l|}{ Propriedades } & $\mathrm{X}$ \\
\hline & \multicolumn{3}{|c|}{$\begin{array}{l}\text { Parede básica } \\
\text { Alvenaria de vedacao } \\
\text { vertical - } 150 \mathrm{~mm}\end{array}$} \\
\hline \multicolumn{4}{|l|}{ Paredes (1) } \\
\hline \multicolumn{3}{|l|}{ Fase } & \multirow[t]{3}{*}{$\wedge$} \\
\hline Fase criada & & Construção nova & \\
\hline \multicolumn{3}{|c|}{ Fase demolida Nenhum } & \\
\hline \multicolumn{3}{|c|}{ Outros } & \\
\hline Patologia & & $\square$ & \\
\hline Descrição d & a ... & Estufamento e & \\
\hline Tempo para & a ... & $2160.000 \mathrm{~h}$ & \\
\hline
\end{tabular}

Fonte - Os autores

Figura 7 - Planilha gerada pelo REVIT

\begin{tabular}{|c|c|c|c|}
\hline \multicolumn{4}{|c|}{$<$ Tabela de patologias > } \\
\hline A & B & $\mathrm{C}$ & D \\
\hline Tipo & Patologia & Descrição da patologia & Tempo para manute \\
\hline Alvenaria de vedacao vertical & $\checkmark$ & Colonização de microorganismos & $720 \mathrm{~h}$ \\
\hline Alvenaria de vedacao vertical & $\square$ & Estufamento e deterioração da pintura & $2160 \mathrm{~h}$ \\
\hline
\end{tabular}

Fonte - Os autores 
Dessa forma, o agrupamento dos dados para a gestão da manutenção foram compilados num único arquivo, evitando consulta em múltiplos locais, otimizando o serviço de gerenciamento do empreendimento. Destaca-se, inclusive, o inconveniente na estimativa temporal para a intervenção, onde, a ferramenta, adota "horas" e não "dias" o que poderá comprometer a compreensão entre os profissionais envolvidos.

\section{CONSIDERAÇÕES FINAIS}

O trabalho se desenvolveu através de uma análise exploratória do local estudado sendo identificadas, inicialmente, as anomalias e as causas de sua ocorrência. Devido a inexistência de um projeto em BIM da região em estudo, não foi possível identificar, de imediato, a existência da tubulação hidráulica, o que poderá demandar uma maior acuraria profissional para realização do diagnóstico e proposição das medidas corretivas, sendo que o BIM tende a otimizar os serviços de gestão da manutenção.

Deve-se considerar que As Built foi efetuado em um ambiente pequeno, o que ocasionou reduzida exploração da ferramenta, embora tenha se mostrado eficaz para a representação do projeto, possibilitando constatar sua real contribuição em grandes projetos, onde todo o empreendimento é idealizado com o BIM. Dessa forma, os prováveis agentes causadores de manifestações patológicas serão identificados imediatamente, bem como a previsão possíveis falhas no sistema construtivo. Os projetos em BIM (e suas ferramentas) são aliados importantes da manutenção de edificações, uma vez que possibilitam o desenvolvimento e execução adequada da manutenção preventiva.

Cabe ressaltar ainda que o método GUT, utilizado para estabelecer o Plano de Manutenção, auxilia a estabelecer diretrizes na elaboração da manutenção do empreendimento do As Built, segundo uma ordem de priorização, tornando-se um instrumento adequado para ser utilizado junto com o BIM nos serviços manutenção das edificações. Para isso, foram associadas as informações coletadas no software BIM, gerando uma planilha que concentra os dados dos sistemas da edificação que necessitam de intervenção incluindo o prazo de intervenção.

Por fim, deve-se considerar que o mapeamento de danos (representação gráfica das manifestações patológicas), que sintetiza as informações acerca do estado de conservação geral do edifício e suas partes, é também uma importante ferramenta para complementação do diagnóstico e das atividades a serem realizadas em campo, devendo estar comtemplado na Gestão da Manutenção.

\section{AGRADECIMENTOS}

Ao Conselho Nacional de Desenvolvimento Científico e Tecnológico (CNPq).

\section{REFERENCIAS}

ASSOCIAÇÃO BRASILEIRA DE NORMAS TÉCNICAS. NBR 5674: Manutenção de Edificações - Procedimento. Rio de Janeiro, 2012.

NBR 14645: Elaboração do "como construído" (as built) para edificações Procedimento. Rio de Janeiro, 2011.

AL-ASHMORI, Y. Y; OTHMAN, I.; RAHMAWATI, Y.; AMRAN, Y. H. M.; SABAH, S. H. A.; RAFINDADI, A. D.; MIKIC, M. Bim benefits and its influence on the BIM implementation in Malayysia. Ain Shams Engineering Journal, 2020. 
CARVALHO, A.; SILVA, I.; VICENTE, R.; BARBOSA, M. T. O emprego do método GUT na priorização da solução de manifestações patológicas. Estudo de caso: Instituto Maria. In: CONGRESSO INTERNACIONAL NA RECUPERAÇÃO, MANUTENÇÃO E RESTAURAÇÃO DE EDIFÍCIOS, 5., 2020, Rio de Janeiro. Anais [...] Rio de Janeiro: 2020.

DE OLIVEIRA, Gustavo Aparecido. Proposta de Manual para desenvolvimento de projetos em BIM através do método Design Science Research. Dissertação (Mestrado em Ciências) - Escola Politécnica da Universidade de São Paulo, São Paulo, 2019.

EASTMAN, C. TEICHOLZ, P.; SACKS, R.; LISTON, K. BIM HANDBOOK: A guide to Building Information Modeling for owners, managers, designers, engineers, and constractors. Hobojen, Nova Jérsi, EUA: John Wiley \& Sons, 3 ed., 2018.

Faculdade de Engenharia da Universidade Federal de Juiz de Fora. Disponível em: https://www.ufjf.br/engenharia/institucional/historia/

FONTAIN, J.; LANGAR, S. Building Information Modeling (BIM) outsourcing among general contractors. Automation in Construction, v. 95, p. 107-117, 2018.

HAM, N.; MOON, S.; KIM, J. H.; KIM, J. J. Optimal BIM staffing in construction projects using a queueing model. Automation in Construction, v. 113, 2020.

PENTTILÄ, H. Describing the changes in architectural Information Technology to understand design complexity and free-form architectural expression. Journal of Information Technology in Construction, v. 11, Special Issue, p. 395-408, 2006.

PINTO; NASCIF, A.K. e Júlio. Manutenção: Função Estratégica. $4^{\circ}$ Edição. Rio de Janeiro. Qualitymark. 2012.

SANTOS, K.; CALMON, J. L.; ALVES, L. O EMPREGO DO BIM COMO FERRAMENTA DE SUPORTE à GESTÃO DA MANUTENÇÃO DE EDIFICAÇÕES. ln: CONGRESSO BRASILEIRO DE PATOLOGIA DAS CONSTRUÇÕES, Campo Grande, 2018. Anais [...] Campo Grande, 2018.

SENA, T. C. de; SENA, P. C. P. de; PHILIPPSEN JR., L. A; FABRICIO, M. M.; PRATSCHKE, A. 5d para Simulação e Tomada de Decisão no Processo de Projeto de Edificações. In: ENCONTRO NACIONAL DE TECNOLOGIA DO AMBIENTE CONSTRUÍDO, 17., 2018, Foz do Iguaçu. Anais [...] Porto Alegre: ANTAC, 2018.

SOTILLE, M.A. GUT - Gravidade, Urgência e Tendência. PM Tech Capacitação em Projetos. 2014.

SOUZA, V. C.; RIPPER, T. Patologia, recuperação e reforço de estruturas de concreto. São Paulo: Pini, 1998.

SUCCAR, B.; SHER, W.; WILLIAMS, A. Measuring BIM performance: five metrics. Architectural, Engineering and Design Management, v. 8, n. 2, p. 120-142, 2012.

VALERO, E.; FORSTER, A.; BOSCHÉ, F; HYSLOP, E.; WILSON, L.; TURMEL, A.; Automated defect. Detection and classification in ashlar masonry walls using machine learning. Automation in Construction, v. 106, 2019. 